Meta

Journal des traducteurs

Translators' Journal

\title{
Le cinéma américain et la langue française
}

\section{Geneviève Petiot}

Volume 32, numéro 3, septembre 1987

La fertilisation terminologique dans les langues romanes

URI : https://id.erudit.org/iderudit/003829ar

DOI : https://doi.org/10.7202/003829ar

Aller au sommaire du numéro

Éditeur(s)

Les Presses de l'Université de Montréal

ISSN

0026-0452 (imprimé)

1492-1421 (numérique)

Découvrir la revue

Citer cet article

Petiot, G. (1987). Le cinéma américain et la langue française. Meta, 32(3),

299-305. https://doi.org/10.7202/003829ar d'utilisation que vous pouvez consulter en ligne.

https://apropos.erudit.org/fr/usagers/politique-dutilisation/ 


\section{LE CINÉMA AMÉRICAIN ET LA LANGUE FRANÇAISE}

GENEVIÈve PETIOT

Sorbonne Nouvelle, Paris, France

L'étude présentée ici est plus modeste que le titre annoncé pour cette communication. En effet, sans examiner, d'un point de vue sociolinguistique, les raisons et le fonctionnement socio-économique de l'importance du cinéma américain dans le monde du film, je me limiterai à la description du fonctionnement des termes cinématographiques français, tel qu'on peut l'étudier dans les textes, oraux et écrits, de langue française. C'est à partir de leurs occurrences textuelles variées ${ }^{1}$, puisque le corpus regroupe aussi bien des articles journalistiques plus ou moins spécialisés, que des ouvrages d'études (livres et revues), et des dictionnaires de langue, et de cinéma, que j'ai étudié les différents composants lexicaux, et les modalités d'assimilation dans la langue, en ce qui concerne les emprunts anglo-américains, ainsi que les résistances à une intégrationnormalisation. Les listes terminologiques officielles ${ }^{1}$ ont été consultées à cette fin, dans un second temps seulement, pour une approche comparative des termes recommandés et des termes rencontrés.

Il s'agit donc ici d'une description de fonctionnement lexical en discours, apparentée aux études lexicologiques ${ }^{2}$.

\section{I - LE VOCABULAIRE CINÉMATOGRAPHIQUE FRANÇAIS}

tel qu'on peut le recenser en dictionnaire, et tel que l'on le rencontre dans ses réalisations textuelles.

\section{LE DOMAINE CINÉMATOGRAPHIQUE ${ }^{3}$}

Il forme un ensemble complexe, constitué par les relations qu'il entretient avec des domaines techniques et technologiques ${ }^{4}$, langagiers et socioculturels, distincts de lui, mais auxquels il emprunte des moyens et/ou avec lesquels il partage certaines caractéristiques et certains types de fonctionnement. Sa spécificité se définit par ces interactions et interrelations.

Ainsi, à l'optique il prend notamment appareils, émulsions, supports... et les dénominations constituées, comme au domaine de la phonation-enregistrementreproduction il prend techniques et termes. Ajoutons les relations du cinéma et de la télévision, relations techniques, professionnelles et terminologiques.

De plus, en tant qu'" art du spectacle ", il hérite de toute une terminologie propre au théâtre, mais aussi à la littérature, à la musique, à l'image... Mise en scène, décors, costumes, maquillages, acteurs, rôles, affiches,... pour n'indiquer que des " rubriques" générales, seront donc des termes d'une koiné non spécifique du cinéma. Nourrissant aussi études, critiques, ouvrages diversifiés, il relèvera d'approches rhétoriques, narratives, en particulier, elles aussi formant des domaines terminologiques.

À l'image de ce domaine complexe, car défini comme domaine "en contact ", on trouvera une terminologie composite, formée d'unités étrangères ou françaises, mettant en concurrence des termes plus ou moins spécifiques. Ainsi, fonctionneront en équivalence sémantique "metteur en scène, réalisateur, directeur d'acteurs", par exemple. 


\section{LES PROCÉDÉS LINGUISTIQUES DE CONSTITUTION LEXICALE}

On retrouve là le problème général de la "néologie ", en tant que "processus de production au sein d'une langue des formes lexicales nouvelles 5 ». Des premières caractéristiques signalées précédemment, et du statut du terme technique ainsi présenté par L. Guilbert ${ }^{6}$ : "Le terme technique tend à être monosémique ou plutôt monoréférentiel dans chaque domaine particulier de la connaissance... " "Le terme scientifico-technique ne peut être dissocié de sa fonction sociale, de la personnalité du locuteur spécialiste ", vont découler des " tensions " discursives et des résistances à la "normalisation " signalées plus loin.

\subsection{L'emprunt}

"solution la plus évidente, la plus paresseuse ${ }^{7}$ ", est d'autant plus courant que nombre d'objets techniques sont dénommés par des termes anglo-américains (même si ces mêmes objets sont principalement inventés et fabriqués au Japon!) Les emprunts sont donc nombreux, et sont d'âge différent. Citons : travelling, off (voix -, son -), flashback, perchman, rush(es), vamp, star, remake, zoom, cameraman...

Pour mémoire, rappelons que vedette est un terme emprunté à l'italien, mais que cet emprunt pleinement naturalisé ne partage plus avec les exemples cités cette caractéristique de "dénomme[r] la notion et connote[r] son origine ${ }^{7}$ ».

2.2 Les " néologismes ordinaires ${ }^{8}$ »

associant un signifiant nouveau et un signifié nouveau, dénommant le référent, sont très peu nombreux. On peut citer : empâtage, qui ne figure pas dans le Petit Robert du corpus, formé sur em-pât(er, -ement), avec le suffixe -âge, et ainsi défini : " lors des opérations de développement, application d'un révélateur pâteux sur la piste sonore optique d'un film en couleurs ${ }^{9}$ "; et vedettariat, qui ne figure pas lui non plus dans le Petit Robert du corpus.

\subsection{Les " néologismes de sens 8 "}

associant à un signifiant déjà existant un signifié nouveau. Ce procédé est appelé par $\mathbf{A}$. Rey " emprunt interne terminologique ${ }^{7}$ » et participe soit au phénomène de l'homonymie, soit à celui de la polysémie. Pour le premier cas, on relève charte : " tableau, filmé en fin de prise, comportant deux séries standardisées de plages colorées et de plages grises, et qui permet au laboratoire de contrôler le rendu des couleurs " ", découverte, " toile peinte ou photographiée placée derrière une ouverture de décor de studio (une fenêtre par ex.) pour simuler l'arrière-plan ${ }^{9}$ » en relation avec les décors de théâtre ; en revanche champ ou plage semblent se situer à l'intersection des deux catégories sémantiques.

Le phénomène de la polysémie est le plus productif : tourner, tournage, (cf. poterie) ; monter, montage, monteur/-euse ; tirage, tireuse ; coller, collage, colleuse, collure ; doubler, doublage, doubleur/-euse ; écran ; panoramique, panoramiquer ; générique ; réalisateur ; régisseur ; plateau...

On peut ajouter à cette liste non exhaustive rush, signalé dans le Petit Robert comme un terme sportif (emprunté).

De ce point de vue donc, le cinéma n'a pas un fonctionnement lexical original ; il recourt aux différents procédés linguistiques recensés, qui caractérisent le phénomène de la néologie; il n'échappe pas à la règle commune. 


\section{II - LES MODALITÉS D'ASSIMILATION DANS LA LANGUE FRANÇAISE}

Là encore les exemples cités n'épuiseront pas la liste des vocables rencontrés ; ils se contenteront d'illustrer des types de fonctionnement bien connus.

\section{AU NIVEAU PHONOLOGIQUE}

Cette rubrique ne concerne que les emprunts, les néologismes ordinaires et de sens étant produits au sein des séquences phonologiques (les schèmes) du français. vamp entre dans un paradigme : rampe, lampe, harpe ; opposition des consonnes initiales dans le contexte droit $/-$ ãp $/ ; / \mathrm{v} /, / 1 /, / R /$ s'opposent entre elles, et à l'absence de consonne initiale dans /ãp/.

rush entre dans le paradigme riche, ruche, rêche; dans le contexte consonantique gauche $/ \mathrm{R} /$, et droit $/ \mathrm{S} /$, opposition des voyelles $/ \propto /, / \mathrm{i} /, / \mathrm{y} /, / \varepsilon /$.

Contentons-nous aussi de rappeler que la réalisation phonique française des finales -ing et des mots comme parking, camping, travelling, est éloignée de la réalisation anglaise, à l'instar du célèbre bifteck.

\section{AU NIVEAU MORPHOLOGIQUE}

Des séries fonctionnent pour les trois rubriques distinguées en 1 .

panoramique (adjectif) devient substantif, sur lequel se crée le verbe panoramiquer pâte, ..., empâtage

vedette, vedettariat (cf. secrétariat ...)

vamp, vamper (verbe transitif)

zoom, zoomer (verbe intransitif)

De telles séries, conformes aux types morphologiques de néologismes linguistiques, sont distinctes de séries ressenties comme "étrangères ";

perchman, cameraman, tennisman, superman ; au niveau phonologique, en revanche, cette série fonctionne comme celle de toxicomane, cocaïnomane, même si sémantiquement, -man, -mane, tous deux [+ Animé] sont distincts.

Des doublets fonctionnent : lexie complexe : joueur de tennis, tennisman ; et perche comme caméra (emprunt) sont ressentis comme des mots "français"; la liste officielle $^{1}$ remplace perchman par perchiste. En ce cas pourquoi ne pas étendre le procédé à cameraman pour lui substituer cameriste, mot français disponible ; son emploi relevant du néologisme de sens, là où perchiste relève de la polysémie (cf. terme sportif).

Autre série : star, starlette, dont la finale -ette fonctionne comme suffixe diminutif, conforme au modèle fille, fillette.

\section{AU NIVEAU SYNTAXIQUE}

On constate que les emprunts, des plus anciens aux plus récents, voire contemporains, se comportent conformément aux modèles français. En syntagme nominal (S N), ils sont intégrés à la structure Prédéterminant + Nom, et son affectés des catégories du genre et du nombre :

un travelling, des rushes, ...

Ils entrent dans des syntagmes à expansions :

un travelling latéral, le travelling optique, de nombreux zooms,

et sur le modèle de la marche avant, la marche arrière, on trouve :

le travelling arrière, le travelling avant,

modèle distinct de celui de l'assistant camera. Le problème d'un tel syntagme est aussi celui posé par l'emploi du terme caméra, par rapport à la séquence : assistant photographe (caméra : [- Animé] ; photographe : [+ Animé $]^{10}$. 


\section{AU NIVEAU GRAPHIQUE}

C'est là que les résistances à l'assimilation sont les plus fortes, étant donné le fonctionnement et le rôle de l'orthographe française, abondamment étudiés et caractérisés.

La règle sera donc le maintien de la graphie d'origine, qui contribue au rôle connotatif de l'emprunt signalé par A. Rey. Ainsi en va-t-il des finales en -ing, travelling, zooming,..., de vamp, alors que la graphie vampe serait d'autant plus possible que la finale -e a prévalu pour scripte, subtitué à script-girl, faux emprunt pour continuity girl.

La graphie d'origine de star se maintient, alors que starlette est francisé graphiquement. Quant au star-system, il se graphie indifféremment tel quel ou star-système et combine donc les deux possibilités.

Contentons-nous ici de rappeler que le problème de l'orthographe ne peut être traité de façon isolée, et que c'est dans ses relations à la morphologie lexicale qu'il convient de l'aborder ${ }^{10}$.

\section{AU NIVEAU LEXICAL}

L'existence de doublets signalés de façon cursive manifeste l'activité langagière et le fonctionnement déjà décrit des vocabulaires techniques et scientifiques ${ }^{11}$.

Les quelques exemples retenus pour illustrer les modes d'assimilation aux différents niveaux de fonctionnement linguistique situent le vocabulaire du cinéma parmi les autres vocabulaires techniques et scientifiques sans que des caractéristiques originales viennent le définir.

\section{III - LES RÉSISTANCES Ȧ LA NORMALISATION}

Elles se manifestent différemment, en fonction des différents cas de fonctionnement observés.

1. Là où l'on a assimilation, et normalisation par la substitution d'un terme recommandé à un autre emprunté :

cameraman, cadreur; perchman, perchiste ; travelling-matte, cache mobile; zoom, travelling optique ; script-girl, scripte, secrétaire de plateau ; on constate l'emploi textuel des doublets. Notons toutefois que la francisation de scripte s'est imposée et a éliminé script-girl; en revanche, la secrétaire de plateau, comme son modèle la secrétaire de bureau, est exceptionnelle.

En cas de doublet : forme courante (pour le moment) - forme "normalisée ", on peut constater que lorsqu'il y a coexistence et concurrence d'emploi, il n'y a pas forcément synonymie. Cadreur, par rapport à cameraman, ne recouvre pas le même ensemble signifié-référent et présente une définition sémantique réduite. La même remarque vaut pour rush et épreuve (non rencontré dans le corpus), le second terme étant marqué par sa très grande polysémie. Le risque de " flou " sémantique se profile alors, contrairement aux tendances des termes techniques rappelées plus haut.

2. Plus les termes sont anciens et "installés ", plus les résistances à la normalisation semblent fortes ; cadreur ne figure qu'une seule fois dans le corpus, dans le dossier de presse d'un film sorti en octobre 86, le cas ne se retrouvant pas dans les autres dossiers de presse de la même période.

3.. De plus, pour certains emprunts, on relève une extension d'emplois. Ainsi, dans sa nouvelle présentation qui date du $1^{\mathrm{er}}$ octobre 1986, l'hebdomadaire Télérama propose une rubrique intitulée "RUSHES".

4. Dernier phénomène recensé, la "naturalisation" de termes américains alors que fonctionnent couramment des termes français et/ou francisés. Plusieurs procédés la facilitent. vision.

a) Par exemple, la traduction partielle des génériques, tant au cinéma qu'à la télé- 
Cette traduction partielle se fait par traitement diversifié du générique ; le plus souvent, seul est traduit le générique de début, celui de fin ne l'étant pas. Des exemples "entraînants " de séries télévisées considérées comme "populaires ", (re)diffusées, ceux de Starsky et Hutch et encore de Dallas, en témoignent. Du même coup des termes " récusés " comme cameraman, perchman, semblent imposer leur existence aux téléspectateurs.

b) De nouveaux emprunts semblent aussi se diffuser; citons : starring, co-starring, guest-star, introducing,...

c) L'emploi nouveau en discours de termes américains se constate, en substitution des termes français existants. Il en va ainsi pour : guest, cast, casting,...

La définition des deux derniers est différente; on peut cependant constater qu'ils fonctionnent le plus souvent comme synonymes, et même que le second tend à remplacer le premier. Ainsi en 1980, tous deux sont recensés et définis :

cast "synonyme de distribution",

casting " en France est l'opération consistant à choisir les acteurs parmi les petits rôles, les silhouettes et la figuration $[. . .]^{12}$ ".

Cast disparait du lexique des mots techniques retenus par le Dictionnaire Larousse du cinéma en 1986, et casting est ainsi défini : " (mot anglais pour distribution des rôles). Activité consistant à rechercher des acteurs (en pratique, seconds rôles ou figurants) adaptés aux rôles ".

Le second terme cumule alors les définitions des deux termes préalablement relevés ; notons de plus que cast est immédiatement assimilable, phonologiquement et morphologiquement, au contraire du second, dont la finale "connote son origine étrangère $"$.

Ces emplois nouveaux apparaissent en discours journalistique, et se diversifient en relation à des domaines différents.

Ainsi, dans Télérama (juin 1986), Gérard Depardieu, interrogé, se félicite du «casting" de son dernier film (français).

Dans la Revue du cinéma ${ }^{13}$, Camille de Casabianca déclare : "En plus des trois acteurs principaux, la plupart des comédiens avaient une fonction technique sur le film ; le perchman, la scripte, l'assistant, le directeur de production [...] c'était un casting plutôt étonnant $[. .$.$] "$

Extension d'emploi du terme : dans son Carnet de notes (magazine musical) ${ }^{14}$, Pierre Bouteiller renchérit : "Signalons le casting fabuleux ; il y a Gérard Souzay... ".

On peut relever de ces différents exemples que casting égale cast dans les deux derniers cas ; il désigne une réalité étrangère à la communauté linguistique d'accueil, comme hit-parade pour palmarès, ou shopping pour lèche-vitrines.

Le dernier exemple manifeste de plus le transfert du terme du cinéma à un autre domaine, celui de la musique. Précisons enfin que le domaine musical dont il est question n'est ni le rock, ni le jazz, mais la musique classique.

Ces différents emplois relèvent pleinement du fonctionnement de systèmes connotatifs, et d'une approche sociolinguistique des phénomènes.

d) Dernier mode d'" américanisation " linguistique du cinéma, formant système avec les cas relevés précédemment, celui du traitement des titres.

Outre les traductions des titres d'origine, selon les modèles de " fidélité " et/ou de réalisations originales, un double cas se présente. Celui de la non traduction "Out of Africa", "Love Story",... Mais aussi celui, plus inattendu, d'une traduction " anglaise " du titre anglais original ; ainsi "Cash-Cash ", «Manda's Café " sont les titres français affichés. 
Là encore, c'est bien à des problèmes sociolinguistiques, en lien avec des comportements de groupes sociaux se reconnaissant dans des valeurs socioculturelles manifestées discursivement, que l'on a à faire. De tels comportements ont sans doute beaucoup à voir avec les résistances rencontrées à la "normalisation" française.

Il faut donc distinguer le comportement linguistique et le comportement discursif du vocabulaire du cinéma. Ces deux comportements sont d'ailleurs analogues respectivement à ceux déjà étudiés d'autres domaines techniques et scientifiques. Que l'on se réfère par exemple au vocabulaire des sports, et à son fonctionnement en discours.

Il semble pourtant qu'une politique de "normalisation" puisse difficilement ne pas prendre en compte ces problèmes, surtout si elle se soucie de son propre succès.

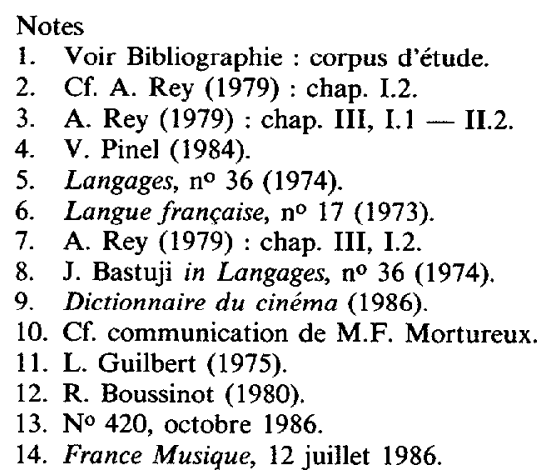

\section{BIBLIOGRAPHIE}

\section{CORPUS D'ÉTUDE : TEXTES PLUS PARTICULIÈREMENT ÉTUDIÉS}

\section{Ouvrages}

AGEL, H. (1962) : l'Esthétique du cinéma, coll. Que sais-je ? no 751, Paris, PUF.

CLAUDE, R. BACHY, V. et B. TAUFOUR (1959) : Panoramique sur le $7^{e}$ art, Paris, Éditions universitaires.

METZ, Ch. (1968) : Essais sur la signification au cinéma, Paris, Klincksieck.

METZ, Ch. (1971) : Langage et cinéma, coll. Langue et langage, Paris, Larousse.

PINEL, V. (1984) : Techniques du cinéma, coll. Que sais-je ?, no 1873, Paris, PUF.

ROPARS-WUILLEUMIER, M.Cl. (1981) : le Texte divisé, Paris, PUM.

SADOUL, G. (1949) : Histoire de l'art du cinéma, Paris, Flammarion.

SADOUL, G. (1962) : le Cinéma français, Paris, Flammarion.

Collection "Anthologie du cinéma ", supplément à l'Avant scène du cinéma, Paris.

Collection " $7 \mathrm{e}$ art ", Paris, le Cerf.

Dictionnaires de cinéma

BOUSSINOT, R. (1980) : l'Encyclopédie du cinéma, Paris, Bordas.

Dictionnaire du cinéma (1986), Paris, Larousse.

\section{Revues}

Change, "Le montage" (1968) : Paris, Seuil.

Communications, no 23 (1975), no 38 (1983), Paris, Seuil.

Fiches du cinéma, Paris, Chrétiens Médias.

La Revue du cinéma, Paris.

Les Cahiers du Cinéma, Paris.

Positif, Paris. 
Rubriques de cinéma des quotidiens le Monde, le Matin, et des hebdomadaires l'Événement du jeudi, le Canard enchaîné, Télérama. Dossiers de presse $(1983,1984,1985,1986)$.

\section{Dictionnaires de langue}

Dictionnaire alphabétique de la langue française (1966) : Paris, Société du nouveau Littré, le Robert, 6 vol. Le Petit Robert (1967) : Paris, Société du nouveau Littré.

Encyclopédie alphabétique Larousse (1977) : Paris, OMNIS, 2 vol.

Larousse de la langue française LEXIS (1977) : Paris, Larousse, 2 vol.

Petit Larousse illustré (1925) : Paris, Larousse.

Arrêté relatif à l'enrichissement du vocabulaire de l'audiovisuel et de la publicité $d u$ 10 octobre 1985.

DOC/PP. 2 décembre 1985, no 49.

\section{LINGUISTIQUE}

\section{Ouvrages}

GUILBERT, L. (1975) : la Créativité lexicale, coll. Langue et langage, Paris, Larousse.

Néologie et lexicologie (1979) : Hommage à Louis Guilbert, coll. Langue et langage, Paris, Larousse.

REY, A. (1979) : la Terminologie noms et notions, coll. Que sais-je ?, $\mathrm{n}^{\circ} 1780$, PUF.

\section{Revues}

Langue française (1978) : "Les vocabulaires techniques et scientifiques ", n 17, Paris, Larousse, février. Langages (1974) : "La néologie lexicale", nº 36, Paris, Didier-Larousse, décembre. 